\title{
Article \\ DNA Methylation Profiling for the Diagnosis and Prognosis of Patients with Nontuberculous Mycobacterium Lung Disease
}

\author{
Jee Youn $\mathrm{Oh}^{1}$, Young Kyung Ko ${ }^{1}$ and Jeong-An Gim ${ }^{2, *}$ \\ 1 Division of Pulmonary, Allergy and Critical Care Medicine, Department of Internal Medicine, \\ Korea University Guro Hospital, Seoul 08308, Korea; happymaria0101@hanmail.net (J.Y.O.); \\ youngsoka@naver.com (Y.K.K.) \\ 2 Medical Science Research Center, College of Medicine, Korea University Guro Hospital, Seoul 08308, Korea \\ * Correspondence: vitastar@korea.ac.kr
}

check for updates

Citation: Oh, J.Y.; Ko, Y.K.; Gim, J.-A. DNA Methylation Profiling for the Diagnosis and Prognosis of Patients with Nontuberculous Mycobacterium Lung Disease. Curr. Issues Mol. Biol. 2021, 43, 501-512. https:// doi.org/10.3390/cimb43020038

Academic Editor: Rafael Franco

Received: 14 May 2021

Accepted: 24 June 2021

Published: 28 June 2021

Publisher's Note: MDPI stays neutral with regard to jurisdictional claims in published maps and institutional affiliations.

Copyright: (C) 2021 by the authors. Licensee MDPI, Basel, Switzerland. This article is an open access article distributed under the terms and conditions of the Creative Commons Attribution (CC BY) license (https:/ / creativecommons.org/licenses/by/ $4.0 /)$.

\begin{abstract}
The incidence of nontuberculous Mycobacterium (NTM) lung disease is rapidly increasing; however, its diagnosis and prognosis remain unclear while selecting patients who will respond to appropriate treatment. Differences in DNA methylation patterns between NTM patients with good or poor prognosis could provide important therapeutic targets. We used the Illumina MethylationEPIC (850k) DNA methylation microarray to determine the pattern between differentially methylated regions (DMRs) in NTM patients with good or poor prognosis ( $n=4$ /group). Moreover, we merged and compared 20 healthy controls from previous Illumina Methylation450k DNA methylation microarray data. We selected and visualized the DMRs in the form of heatmaps, and enriched terms associated with these DMRs were identified by functional annotation with the "pathfinder" package. In total, 461 and 293 DMRs ( $\mid$ Log2 fold change $\mid>0.1$ and $P<0.03$ ) were more methylated in patients with four poor and four good prognoses, respectively. Furthermore, 337 and 771 DMRs ( I Log2 fold change $\mid>0.08$ and $P<0.001$ ) were more methylated in eight NTM patients and 20 healthy controls, respectively. TGFBr1 was significantly less methylated, whereas HLA-DR1 and HLA-DR5 were more methylated in patients with poor prognosis (compared to those with good prognosis). LRP5, E2F1, and $A D C Y 3$ were the top three less-methylated genes in NTM patients (compared with the controls). The mTOR and Wnt signaling pathway-related genes were less methylated in patients with NTM. Collectively, genes related to Th1- cell differentiation, such as TGFBr1 and HLA-DR, may be used as biomarkers for predicting the treatment response in patients with NTM lung disease.
\end{abstract}

Keywords: DNA methylation; nontuberculous Mycobacterium; tuberculosis

\section{Introduction}

The incidence and importance of nontuberculous Mycobacterium (NTM) lung disease are rapidly increasing [1,2]. NTM diseases are mainly caused by Mycobacterium avium complex (MAC), Mycobacterium kansasii, and Mycobacterium abscessus strains [1]. NTM is prevalent in people with damaged immune systems and requires prolonged treatment; this treatment is accompanied by various side effects such as severe gastrointestinal distress or hearing loss [3]. The prognosis and outcomes of patients with NTM vary based on the genetic and immunological factors [4-6]; some cases are associated with spontaneous resolution, whereas others are refractory despite 2-3 years of antibiotic treatment [6-9]. Therefore, there is an urgent need to develop tools to diagnose NTM at early stages and to identify patients who will respond to appropriate treatment.

DNA methylation involves the addition of a methyl group to the DNA (frequently at the cytosine-guanine dinucleotide (CpG) sites) [10]. DNA methylation profiles may be altered, and they regulate the expression of genes in response to external or internal conditions. Moreover, these profiles are modified during disease progression; thus, they can enable disease identification. Abnormal DNA methylation induced in response to chronic viral infections enables the viruses to evade the host immune surveillance machinery. DNA 
methylation has also been studied as a biomarker of infection-induced stress [11,12]. The Illumina Infinium MethylationEPIC (EPIC) array enables the simultaneous measurement of the methylation marks at more than 860,000 CpG sites in almost all RefSeq genes [13], thereby enabling the identification of differentially methylated regions (DMRs) between groups. In a previous study, a standard statistical significance for extracting DMR from EPIC chip-derived data was presented; additionally, we tried to extract approximately 1000 DMRs from the two comparison groups [14]. Therefore, in the present study, we used the EPIC array to determine the correlation between DNA methylation profiles and disease progression in NTM. Changes in the DNA methylation patterns of promoters and enhancers regulate gene expression; thus, investigating epigenetic mechanisms at a genome-wide level in humans can bridge the gap between NTM susceptibility and gene expression variation.

An epigenome-wide association study (EWAS) is an additional method used to identify effective epigenetic biomarkers, which can also be used for the identification and prognosis of NTM lung disease. To date, EWAS has been performed to identify altered DNA methylation patterns in several complex diseases, such as diabetes, obesity, schizophrenia, and respiratory diseases [15-18]. DNA methylation patterns have been studied in Mycobacterium tuberculosis infection samples and have been used as biomarkers [19]. Moreover, immune-related factors, used to predict the progression of NTM, have been identified in NTM samples, [20]. Serum from patients with NTM has also been used to detect the association between NTM and other diseases, such as rheumatoid arthritis, bronchiectasis, chronic obstructive pulmonary disease, and cystic fibrosis patients [21]. Nevertheless, to date, no EWAS has been performed for NTM prognosis or to compare the DNA methylation patterns between patients with NTM and healthy controls. Therefore, in the present study, we evaluated the association of the DNA methylation profiles between NTM patients with poor or good prognoses using the EPIC platform, and merged these data with those of a previous DNA methylation study on healthy controls without any respiratory diseases using the Illumina 450k methylation array. Furthermore, we conducted a comprehensive bioinformatic analysis of the DNA methylation patterns between the two data sets and identified and visualized the DMRs. Based on these DMRs, enriched terms, such as Thelper (Th)-cell differentiation, mitophagy, spliceosome, adherens junction, diseases, or cancer (e.g., breast cancer, thyroid cancer, and chronic myeloid leukemia), signaling pathways (e.g., Wnt, mTOR, AMPK, notch, and sphingolipid), and cell cycle were selected to depict the upset plots and networks.

\section{Materials and Methods}

\subsection{Sample Collection}

Eight patients with NTM, from the Division of Pulmonology, Department of Internal Medicine, Korea University Guro Hospital, were enrolled in this study. This study was approved by the Institutional Review Board of Korea University Guro Hospital (2017GR0012). The patients agreed to provide blood and clinical data with informed consent. All investigations were conducted in accordance with the principles of the Declaration of Helsinki. The biospecimens and data used for this study were provided by the Biobank of Korea University Guro Hospital, located in Korea.

NTM was diagnosed in accordance with the official ATS/IDSA statement [22]. NTM prognosis was classified as good or bad based on the treatment outcome definitions proposed in an NTM-NET consensus statement [6]. Patients with cured disease were grouped as having a good prognosis, whereas those with treatment failure were classified as having a poor prognosis.

\subsection{DNA Extraction and Methylation Microarray}

DNA was isolated from all eight whole blood samples using the BioRobot EZ1 (Qiagen) system according to the manufacturer's instructions. We provided $1.0 \mu \mathrm{g}$ of extracted DNA to Macrogen, Inc. (Seoul, South Korea) for methylation microarray analysis. DNA quality 
control was confirmed using the Infinium FFPE QC Kit (Illumina, San Diego, CA, USA), and DNA restoration was performed using the Infinium HD FFPE DNA Restore Kit (Illumina). Bisulfite conversion was performed using the EZ-96 DNA Methylation Kit (Zymo Research, Irvine, CA, USA), and a methylation microarray was performed using the Infinium MethylationEPIC BeadChip Kit (Illumina, USA). The iScan system (Illumina) was used to read the BeadChips.

Array data were exported, processed, and analyzed using Illumina GenomeStudio version 2011.1 (Methylation Module version 1.9.0) and R version 4.0.3. Each methylation data point was represented by fluorescent signals from methylated $(\mathrm{M})$ and unmethylated (U) alleles. Thereafter, the ratio of fluorescent signals was computed from two alleles as $\beta=(\max (\mathrm{M}, 0)) /(|\mathrm{U}|+|\mathrm{M}|+100)$. Raw $\beta$-values were extracted as $865,918 \mathrm{CpGs}$. Furthermore, background correlations and dye bias equalization were made using the lumi package in R. Beta-mixture quantile normalization was performed to reduce the assay bias using the BMIQ package in R.

\subsection{Merged Public Data Set}

We used publicly available DNA methylation data from the Illumina 450k methylation array based on samples from 446 people included in the Korean Genome and Epidemiology Study Ansan-Ansung (KoGES-ASAS) [23]. Twenty healthy controls were included in this cohort. Sex, age, and BMI crucially affected the NTM diagnosis and prognosis. Therefore, we matched the healthy controls and patients based on age, sex ratio, and BMI, and selected individuals without any respiratory diseases. We further performed ANCOVA to adjust the age, sex, BMI, and underlying diseases for epigenetic markers. Probes common to the $450 \mathrm{k}$ and EPIC datasets were combined using the R "merge" function, and the ComBat method was employed to adjust for the batch effects.

\subsection{Bioinformatic Analysis and Visualization}

To analyze and visualize the characteristics between the two groups, we used the $\mathrm{R}$ version 4.0.3 package. Differences between the groups were identified using a $t$-test and visualized using a volcano plot. I Log2 fold change I and $P$-value were defined as the thresholds, which were adjusted according to the DMR patterns between the two groups. The heatmap and hierarchical clustering plot were constructed using the pheatmap package in R. Volcano plots of DMRs were obtained using the plot function in R with $\log 2$ fold change on the $\mathrm{x}$-axis and the transformed $-\log 10 P$-values on the $\mathrm{y}$-axis. The $\mathrm{R}$ package "pathfinder" was used to select terms enriched by the identified DMRs, and these enriched terms were depicted using upset plots and networks with the "Upset_plot" and "term_gene_graph" functions, respectively [24].

\section{Results}

\subsection{Clinical Characteristics}

The clinical characteristics of the study groups are summarized in Table 1. All eight patients with NTM, aged 42-78 years and who underwent treatment, were infected with the same subtype (MAC). The mean age was similar in the two groups (59.1 \pm 11.2 in the NTM group and $65.5 \pm 5.4$ in the healthy control group); moreover, males comprised $62.5 \%$ of the patients in the NTM group and $65 \%$ in the healthy control group. Patients with NTM exhibited a relatively low body mass index $\left(B M I=19.7 \pm 3.7 \mathrm{~kg} / \mathrm{m}^{2}\right)$; therefore, we matched these to patients in the healthy control group who had a similar BMI (mean $\left.20.5 \pm 1.0 \mathrm{~kg} / \mathrm{m}^{2}\right)$. 
Table 1. Baseline characteristics of patients with NTM and healthy controls.

\begin{tabular}{ccc}
\hline & $\begin{array}{c}\text { NTM } \\
n=8\end{array}$ & HC \\
& $59.1 \pm 11.2$ & $65.5 \pm 5.4$ \\
\hline Age & $5(62.5)$ & $13(65)$ \\
\hline Sex, male & $19.7 \pm 3.7$ & $20.5 \pm 1.0$ \\
\hline BMI $\left(\mathrm{kg} / \mathrm{m}^{2}\right)$ & $2(25)$ & $8(40)$ \\
\hline Smoker & $2(25)$ & $3(15)$ \\
\hline Alcohol & $1(12.5)$ & $0(0)$ \\
\hline Comorbidities & $2(25)$ & $0(0)$ \\
\hline HTN & $2(25)$ & $0(0)$ \\
\hline DM & $0(0)$ & $0(0)$ \\
\hline COPD/Asthma & & \\
\hline Heart failure & & \\
\hline
\end{tabular}

Notes: Data are presented as mean \pm standard deviation for continuous variables and number (\%) for categorical variables. Abbreviations: BMI, body mass index; $\mathrm{COPD}$, chronic obstructive pulmonary disease; $\mathrm{CKD}$, chronic kidney disease; DM, diabetes mellitus; HTN, hypertension; HC, healthy controls; NTM, nontuberculous Mycobacterium.

\subsection{Identification of DMRs According to NTM Prognoses and between Patients and Healthy Controls}

Using $\mid \log 2$ fold change $\mid>0.1$ and $P$-value $<0.03$ as the threshold, we visualized DMRs between NTM patients with good and poor prognoses ( $n=4$ per group) using the "pheatmap" R package. In total, 754 DMRs were identified, including 461 and 293 DMRs that were more methylated in patients with poor and good prognoses, respectively. DMRs are listed according to their $\beta$-values and are depicted as a heatmap in Figure 1a; furthermore, all DMRs are plotted as volcano plots in Figure 2a.

Using | Log2 fold change $\mid>0.08$ and $P$-value $<0.001$ as thresholds, we identified 1108 DMRs between patients with NTM and healthy controls, including 337 and 771 DMRs that were more methylated in eight NTM patients and 20 healthy controls, respectively. The DMRs based on the $\beta$-values of eight patients with NTM and the batch effect-adjusted $\beta$-values of the 20 healthy controls are illustrated in the heatmap in Figure 1b; all DMRs are plotted as volcano plots in Figure 3A.

Using these two different DMR analyses, we identified the top three target regions for DMRs between four NTM patients with poor prognosis and four NTM patients with good prognosis (Table S1), as well those for DMRs between eight patients with NTM and 20 healthy controls (Figure 4). Among the top three probes between the two groups of NTM prognosis, two were located on human leukocyte antigen (HLA)-related genes, and all these probes were located in the introns of these genes. Considering the other two loci, the probe was located in the transforming growth factor beta receptor 1 (TGFBr1) gene. Among the three probes identified between patients with NTM and healthy controls, two were located in the coding sequences of LDL receptor-related protein 5 (LRP5) and E2F transcription factor 1 (E2F1). Moreover, the intron in the region of adenylate cyclase 3 $(A D C Y 3)$ revealed a less-methylated pattern in patients with NTM (compared to that in the controls). 

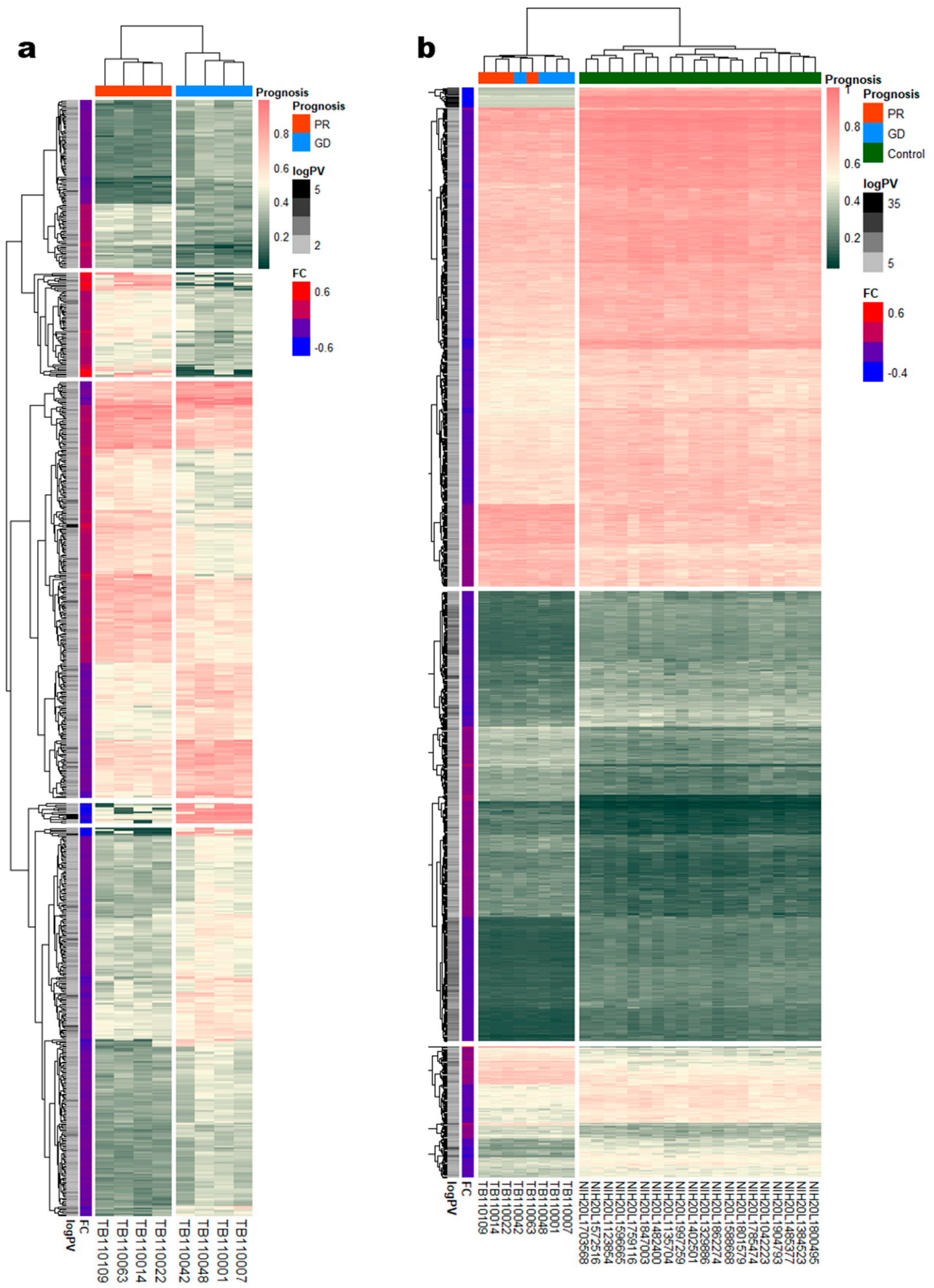

Figure 1. Differential DNA methylation between the NTM prognosis groups and between patients with NTM and healthy controls. (a) Heatmap depicting the fold change in methylation between NTM patients with good or poor prognosis. There were 754 DMRs; the poor prognosis group (red row, PR) accounted for 461 more methylated DMRs, and the good prognosis (blue row, GD) group accounted for 293 more methylated DMRs. Unsupervised clustering of eight samples was observed between the prognosis groups. (b) Heatmap depicting the fold change between patients with NTM and healthy controls. There were 1108 DMRs; the NTM patient (red column annotation bar, PR; blue column annotation bar, GD) group accounted for 337 more methylated DMRs, and the control (green column annotation bar, GD) group accounted for 771 more methylated DMRs. Unsupervised clustering of 28 samples was observed between patients with NTM and the controls. 

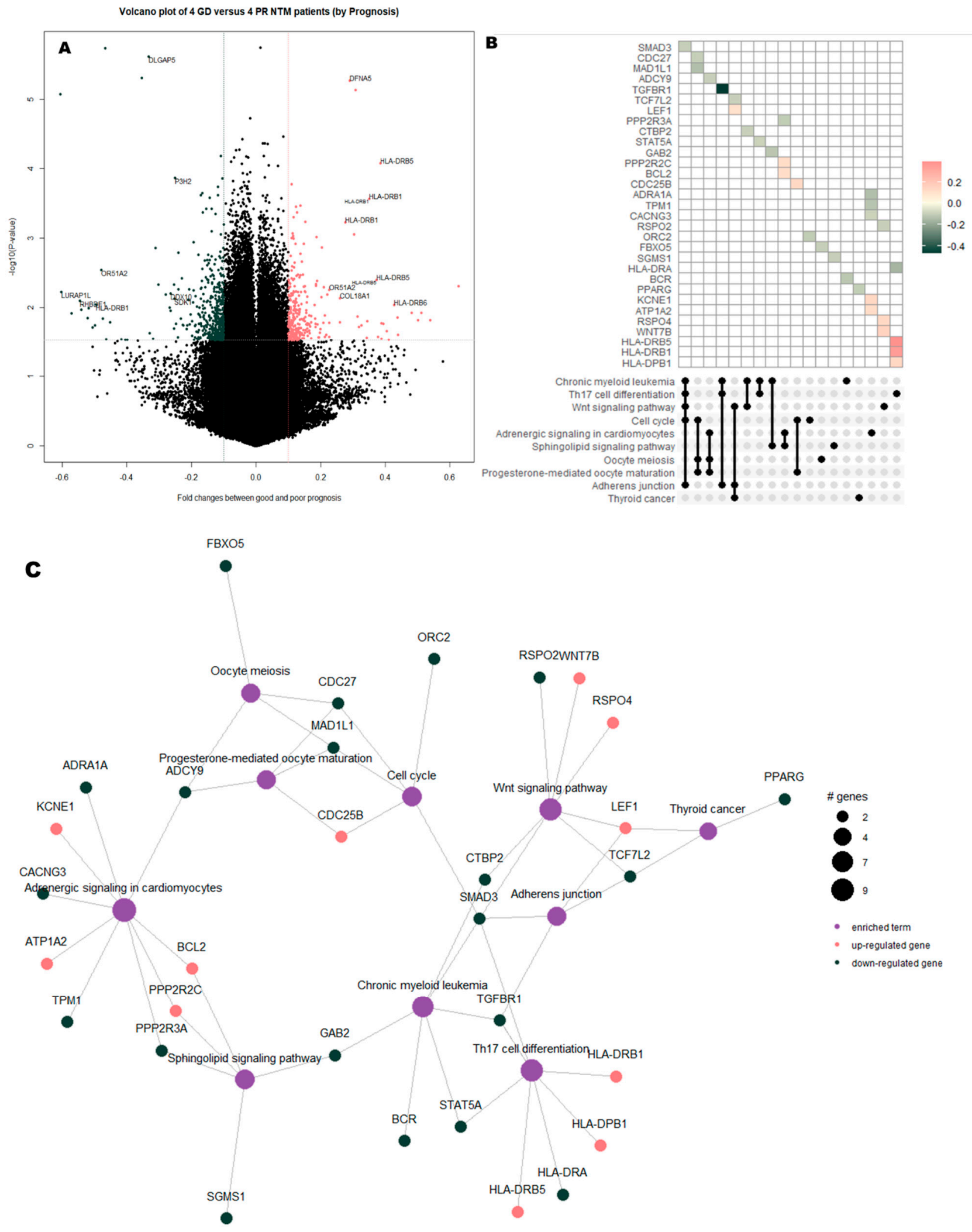

Figure 2. Enriched terms and functional enrichment network of the DMRs between NTM patients with good or poor prognosis. (a) Volcano plot illustrating DMRs (defined as a fold change in methylation $>0.1$ (red) or $<-0.1$ (green) with $P$-value $<0.03$ ) in NTM patients with poor prognosis. (b) Upset plot indicating the methylation patterns of 55 genes with 10 associated enriched terms. (c) Network plot: the color of the circles represents the methylation patterns in patients with NTM, and the size of the enriched circles represents the number of genes involved. 


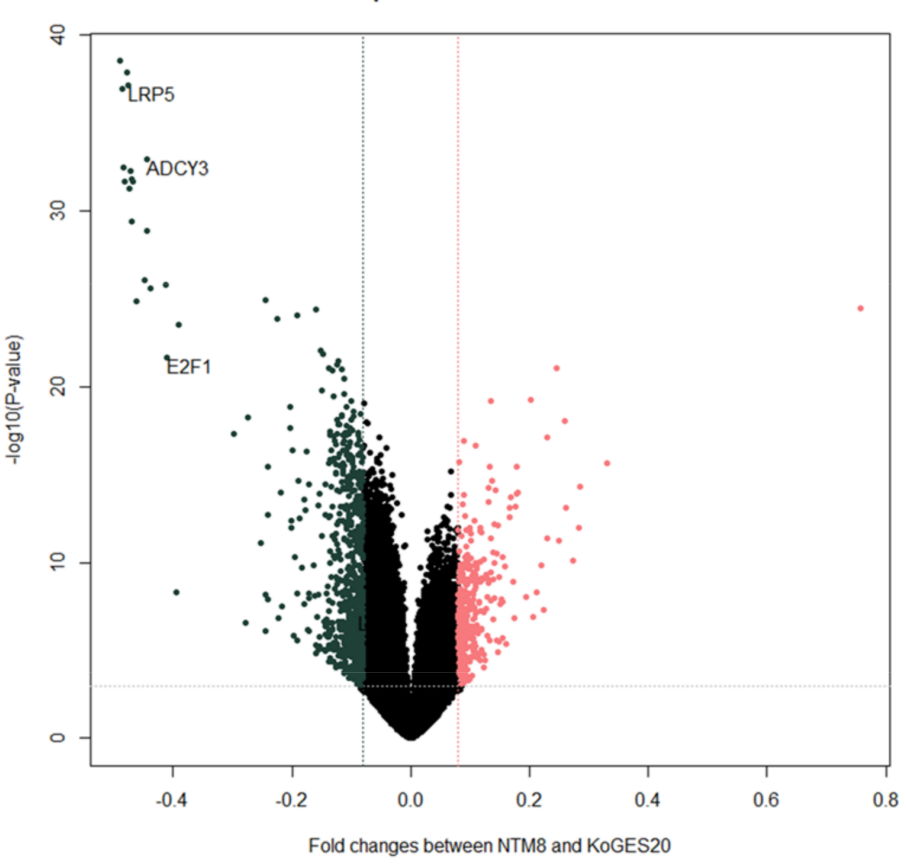

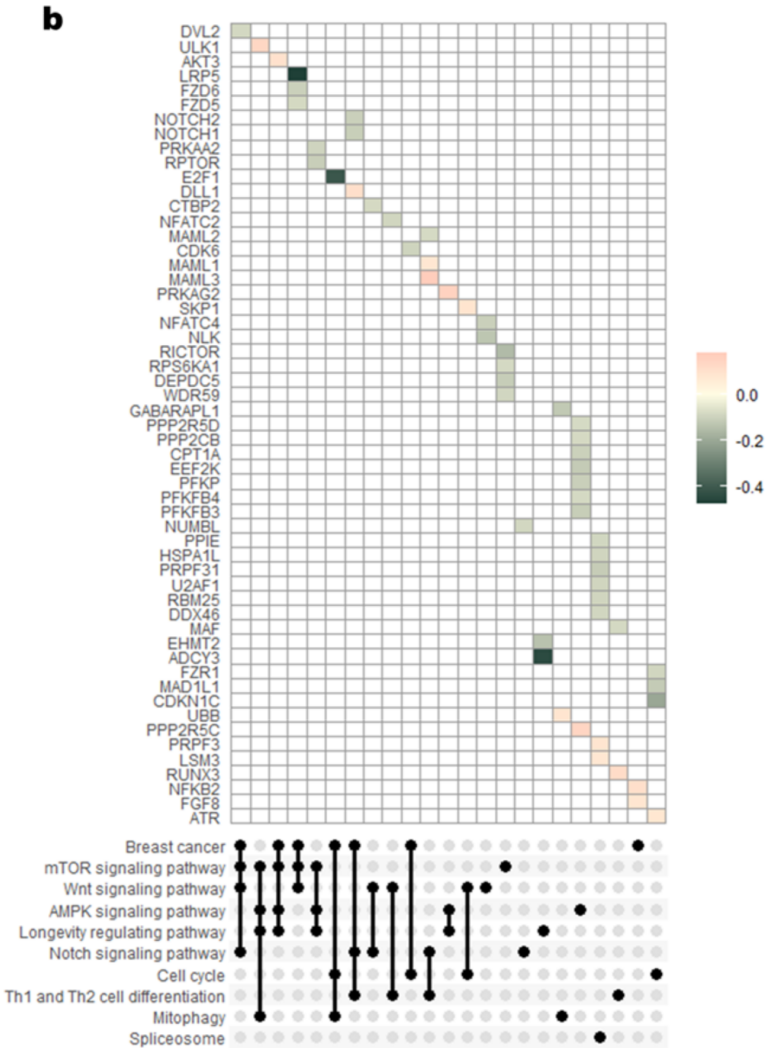
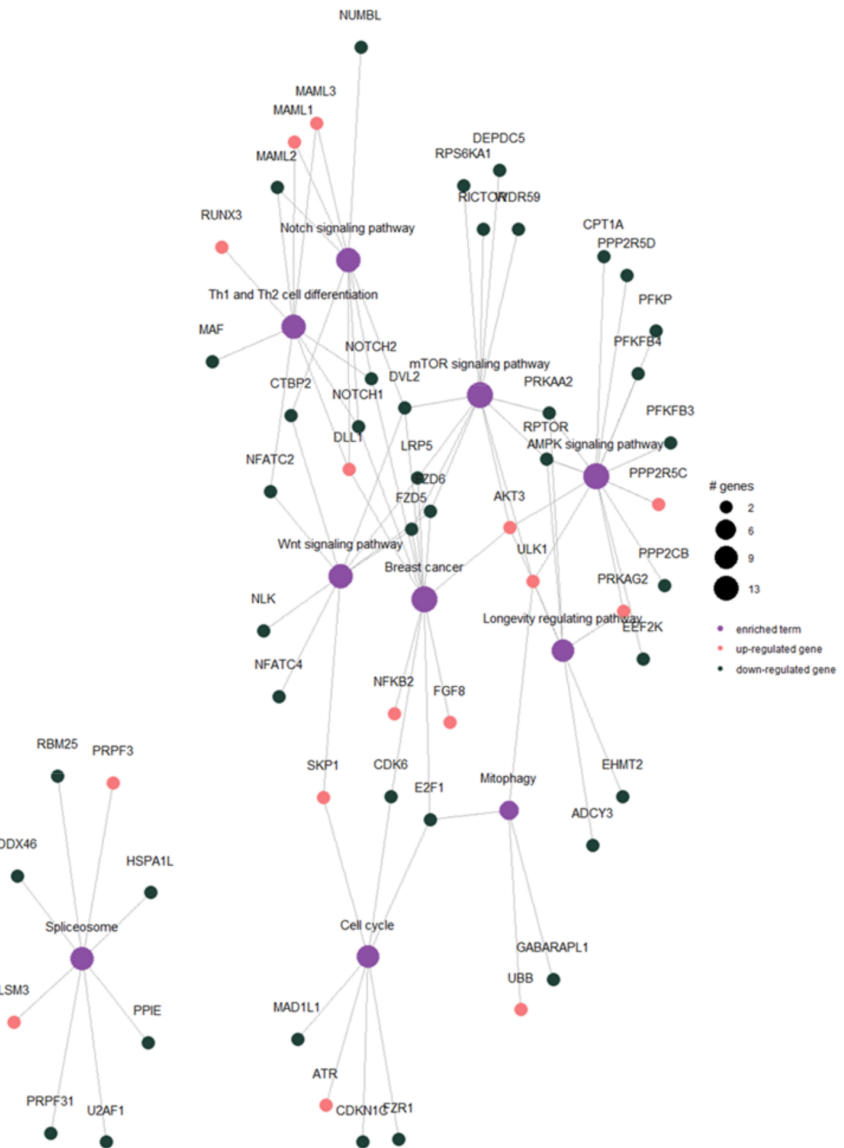

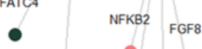
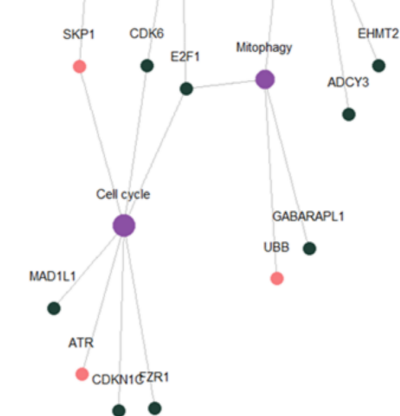

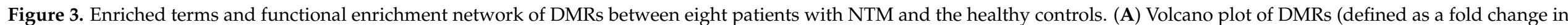

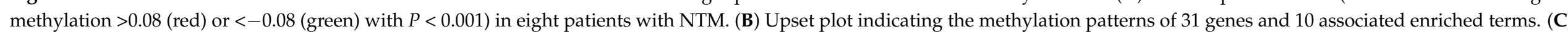

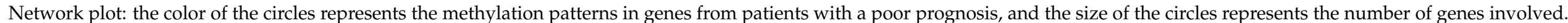


a Boxplot of TGFBr1 (cg13431688) CpG methyl level

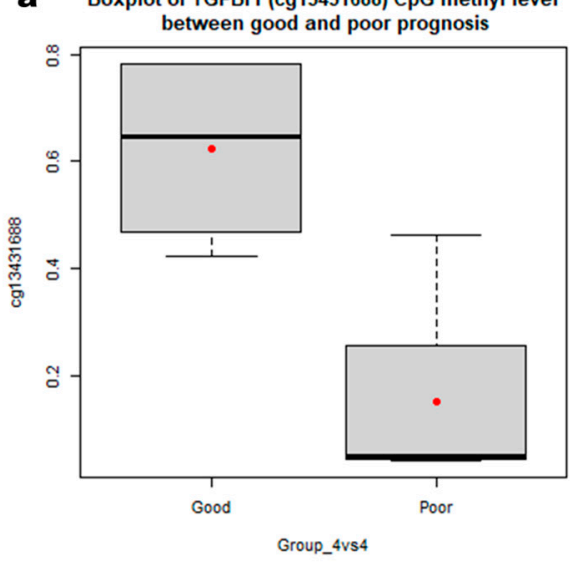

b Boxplot of LRP5 (cg01666550) CpG methyl level

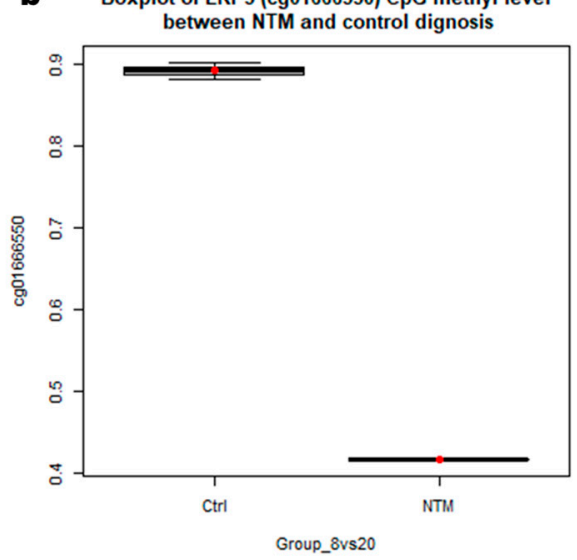

Boxplot of HLA-DRB5 (cg18424635) CpG methyl level
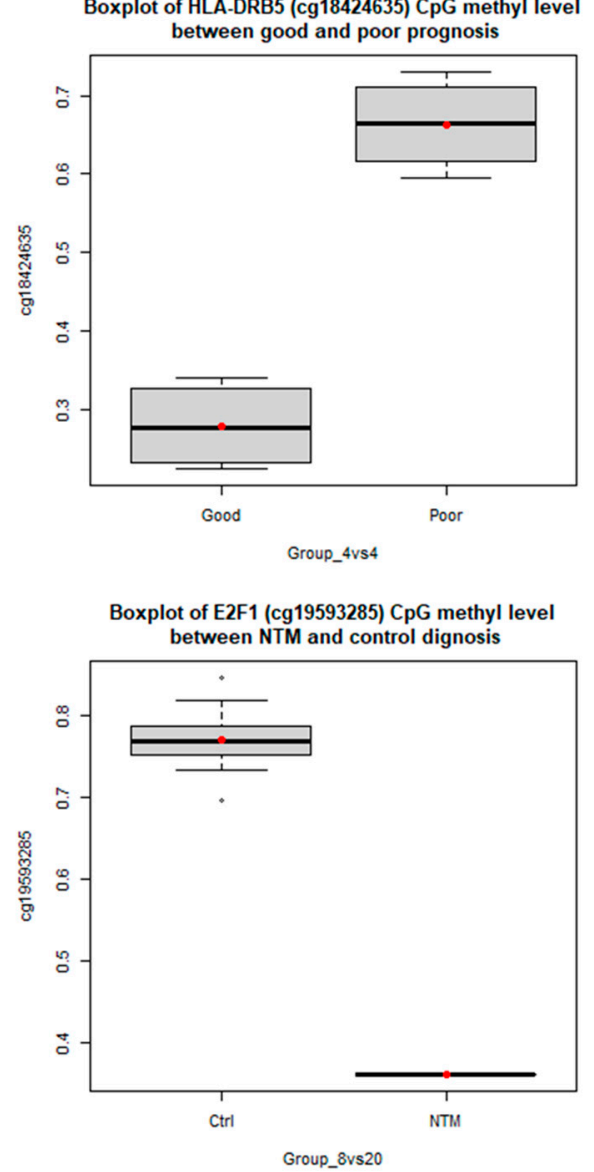

Boxplot of HLA-DRB1 (cg05383619) CpG methyl level

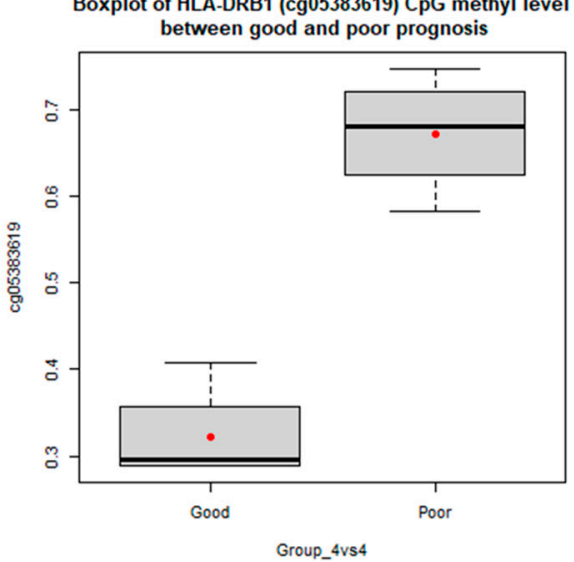

Boxplot of ADCY3 (cg20227471) CpG methyl level between NTM and control dignosis

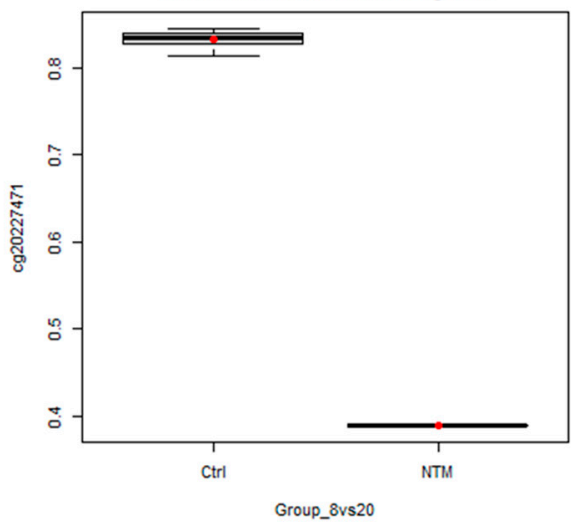

Figure 4. Comparison of the levels of the top three DMRs between (a) NTM patients with good and poor prognoses, as well as (b) between NTM patients and the healthy controls. * ANCOVA was used to adjust the age, sex, BMI, and underlying diseases for epigenetic markers. All $P$-values were $<0.001$.

\subsection{Functional Enrichment Analysis of DMRs}

We visualized the differences between patients with NTM and healthy controls using volcano plots, upset plots, and functional enrichment networks. Identification of genes with similar methylation patterns and their enriched terms may contribute to the gaining of a better understanding of the etiology of NTM. Therefore, we identified common genes or enriched terms while comparing the diagnosis and prognosis. Two genes, mitotic arrest deficient 1 Like 1 (MAD1L1) and C-terminal binding protein 2 (CTBP2), were found to be less methylated in two enriched terms: MAD1L1 was less methylated in the enriched term cell cycle, and CTBP2 was less methylated in the Wnt signaling pathway. Moreover, other genes or enriched terms differed between the two groups under the two respective conditions.

While comparing NTM patients with good or poor prognosis, it was found that HLArelated genes were more methylated in the patients with poor prognosis (Figure 2b,c), and that these genes were enriched for Th17-cell differentiation. Furthermore, Th17 cell differentiation-related genes, particularly TGFBr1, were less methylated in the poor prognosis group. In the network analysis, most of the enriched terms were linked to the less-methylated genes and certain enriched terms, such as Th17-cell differentiation, were linked to more methylated genes in patients with poor prognosis (Figure 2c).

While comparing the eight NTM patients with the 20 healthy controls, LRP5, E2F1, and $A D C Y 3$ were all found to be less methylated in the NTM group (Figure 3B). These genes were enriched in breast cancer cells, as well as in the mTOR, AMPK, and Wnt signaling 
pathways (Figure 3B,C). Most of the enriched terms and genes were linked, except for those in the spliceosome and the eight linked genes. The Notch and AMPK signaling pathways were linked to the more-methylated genes in patients with NTM (Figure 3C).

\section{Discussion}

To the best of our knowledge, this is the first study to evaluate the epigenetic profile of patients with NTM lung disease and to identify novel NTM-related DMRs, which may play pivotal roles in respiratory diseases. The DNA methylation patterns in patients with NTM were clearly distinguishable from those of the healthy controls in terms of the associated genes as well as the enriched terms; moreover, the methylation patterns significantly differed between NTM patients with good or poor prognosis. Presumably, the results and methodology of this study can be used to predict the prognosis of other mycobacterial diseases or to compare it with that in normal subjects. By analyzing the DNA methylation pattern in whole blood, this study provided evidence of a relatively strong prognosis and diagnosis of NTM. The traditional method to detect NTM or TB is time-consuming. The method developed in this study uses a primer to confirm the methylation patterns in many patients. Thus, using this method, NTM or TB can be rapidly and accurately detected (compared to traditional methods); moreover, factors that predict prognosis can also be identified. By confirming the pattern of blood-derived DNA methylation, we can identify disease-related factors underlying the host response to infection.

Numerous identified enriched terms and genes exhibited distinct patterns in NTM patients (compared with the healthy controls) and in NTM patients with poor prognosis (compared with NTM patients with good prognosis). TGFBr1 and HLA-DR-which are related to Th17-cell differentiation-were distinctively methylated in NTM patients with poor prognosis (compared with NTM patients with good prognosis). LRP5—associated with mTOR and Wnt signaling - was significantly less methylated in patients with NTM (compared with the controls). Furthermore, genes associated with the AMPK and mTOR pathways and the cell cycle were found to be related to NTM. As several immune-related genes were less methylated in patients with NTM or in those with a poor prognosis, chronic infection and inflammation with NTM might trigger the cellular transformation of host immune cells [25]. Additionally, some DNA methylation patterns may serve as candidate prognostic markers for immune-related respiratory diseases.

Reciprocal regulation between the AMPK and MTOR pathways plays a pivotal role in mycobacterial diseases [26]. The AMPK pathway regulates host autophagy, mitochondrial biogenesis, and metabolic reprogramming, thereby controlling pathological inflammation in mycobacterial infections [27]. Through this pathway, the host defenses are promoted, leading to enhancement of antimicrobial responses against tuberculosis [26]. Hence, NTM lung disease, which is a mycobacterial infection caused by intracellular pathogens, might also be regulated by the AMPK and mTOR pathways.

In addition to the genes associated with the AMPK and mTOR pathways, MAD1L1 and CTBP2 were found to be commonly less methylated in patients with NTM (compared with the healthy controls) and in patients with a poor prognosis. E2F1—one of the top three DMR probes in NTM - and MAD1L1 control the inflammatory stimulation of the macrophages and contribute to the activation of $\mathrm{T}$ cells in response to the presence of pathogens; these genes are consistently associated with the cell-cycle-enriched term [28]. CTBP2 is associated with the Wnt signaling pathway and plays a crucial role in mycobacterial disease, as it modulates the inflammatory response and controls the adaptive immune response [29-31]. The underlying variations in CTBP2 expression between patients with TB and healthy controls have been identified in other bioinformatic analyses [32].

A previous pathway analysis revealed differential expression of the microRNAs involved in cell growth, migration, and proliferation, and in the Wnt and TGF- $\beta$ signaling pathways in NTM lung diseases [33]. The Wnt-pathway-related gene LRP5 is strongly associated with the progression of pulmonary disease [31], and also regulates the immune mechanisms in TB [30]. This study suggests that the expression of LRP5 may be increased in 
patients with NTM, specifically in those with a poor prognosis, and that DNA methylation may play a crucial role in various immune triggers.

LRP5 is not only involved in the canonical Wnt pathway, but it also regulates the expression of members of the TGF- $\beta$ gene family [31,34]; TGF- $\beta$ has been implicated in the pathogenesis of NTM [35]. TGF- $\beta 1$ suppresses cytokine-induced macrophage activation [36], which may play a crucial role in regulating the immune response against NTM $[37,38]$. TGF- $\beta 1$ is known to downregulate the expression of HLA-DR in macrophages [37], which is consistent with our results. We found that TGFB1 was significantly less methylated, whereas HLA-DR genes were significantly more methylated in NTM; both of these genes were associated with Th17-cell differentiation. Th17 cells induce excessive neutrophilic pulmonary inflammation in MAC [39,40]; moreover, the serum concentrations of Th17-related cytokines reflect the treatment outcome in case of NTM lung disease [41]. Treatment failure in patients with NTM may lead to molecular changes in the Th17 differentiation pathway that is known to be associated with inflammation [41]. In the present study, we found that TGFB1 showed less methylation, and the HLA-DR genes linked with Th17 could act as markers for predicting the treatment outcomes in patients with NTM. Furthermore, genes such as TGFB1 that exhibit somatic mutations in patients with NTM are involved in breast cancer, and NTM infection may act as a potential risk factor for chronic inflammation and cellular transformation; several patients with NTM ultimately developed breast cancer [42]. This might explain why certain genes related to breast cancer are linked to NTM diagnosis.

This study had two limitations. First, cross-validation was not performed for the DMRs presented in this study. Future experiments using more patients with NTM or $\mathrm{TB}$ and cross-validation experiments are required to validate the DMRs or related genes using techniques such as bisulfite sequencing or real-time RT-PCR. Second, NTM patients exhibiting a pattern that differed from the epidemiologically common sex ratio for MAC lung disease were enrolled. MAC lung disease occurs more frequently in women than in men, but its proportion in the present study revealed a different pattern. However, sex differences did not influence the results because epigenetic markers were significant after adjusting for sex. Further studies that take into consideration the sex ratio for MAC lung disease should be carried out. By overcoming the aforementioned limitations in future studies, we look forward to identifying DNA methylation biomarkers that can explain infectious respiratory diseases such as NTM or TB.

\section{Conclusions}

We evaluated the epigenetic profiles for the diagnosis and prognosis of NTM. TGFBr1 was significantly less methylated, whereas $H L A-D R 1$ and $H L A-D R 5$ were more methylated in patients with a poor prognosis (compared to those with a good prognosis). LRP5, E2F1, and $A D C Y 3$ were the top three less-methylated genes in patients with NTM (compared to the controls). Collectively, TGFBr1 and HLA-DR may be used as potential biomarkers for predicting the treatment response in patients with NTM lung disease.

Supplementary Materials: The following are available online at https:/ / www.mdpi.com/article/10 .3390 / cimb43020038/s1. Table S1: CpGs located in the top 11 differently methylated regions between patients with NTM with a good prognosis and poor prognosis, and the 10 differently methylated regions between patients with NTM and healthy controls.

Author Contributions: Conceptualization, J.Y.O. and J.-A.G.; methodology, J.-A.G.; validation, Y.K.K.; formal analysis, J.Y.O.; investigation, J.Y.O.; resources, J.Y.O.; data curation, J.-A.G.; writingoriginal draft preparation, J.Y.O. and J.-A.G.; writing-review and editing, J.Y.O., Y.K.K., and J.-A.G.; funding acquisition, J.Y.O. and J.-A.G. All authors have read and agreed to the published version of the manuscript.

Funding: This research was funded by the Bio \& Medical Technology Development Program of the National Research Foundation (NRF) funded by the Korean government (MSIT), grant number 2019M3E5D1A01068994; the Basic Science Research Program through the NRF funded by the Ministry 
of Education, grant number NRF-2020R1I1A1A01052701; and a Korea University Grant, grant number K2022911.

Institutional Review Board Statement: This study was approved by the Institutional Review Board of Korea University Guro Hospital (2017GR0012). All investigations were conducted in accordance with the principles of the Declaration of Helsinki.

Informed Consent Statement: Informed consent was obtained from all the subjects involved in the study.

Data Availability Statement: All sequencing data sets presented in this study are available upon request from the corresponding author.

Acknowledgments: The biospecimens and data used for this study were provided by the Biobank of Korea University Group Hospital, Korea.

Conflicts of Interest: The funders had no role in the design of the study; in the collection, analyses, or interpretation of data; in drafting the manuscript; or in the decision to publish the results.

\section{References}

1. Haworth, C.S.; Banks, J.; Capstick, T.; Fisher, A.J.; Gorsuch, T.; Laurenson, I.F.; Leitch, A.; Loebinger, M.R.; Milburn, H.J.; Nightingale, M.; et al. British Thoracic Society guidelines for the management of non-tuberculous mycobacterial pulmo-nary disease (NTM-PD). Thorax 2017, 72, ii1-ii64. [CrossRef]

2. Jeon, D. Infection Source and Epidemiology of Nontuberculous Mycobacterial Lung Disease. Tuberc. Respir. Dis. 2019, 82, 94-101. [CrossRef]

3. Bento, C.M.; Gomes, M.S.; Silva, T. Looking beyond Typical Treatments for Atypical Mycobacteria. Antibiotics 2020, 9, 18. [CrossRef]

4. To, K.; Cao, R.; Yegiazaryan, A.; Owens, J.; Venketaraman, V. General overview of nontuberculous mycobacteria opportunistic pathogens: Mycobacterium avium and Mycobacterium abscessus. J. Clin. Med. 2020, 9, 2541. [CrossRef]

5. Shu, C.-C.; The TAMI Group; Lee, C.-H.; Hsu, C.-L.; Wang, J.-Y.; Yu, C.-J.; Lee, L.-N. Clinical Characteristics and Prognosis of Nontuberculous Mycobacterial Lung Disease with Different Radiographic Patterns. Lung 2011, 189, 467-474. [CrossRef]

6. van Ingen, J.; Aksamit, T.; Andrejak, C.; Böttger, E.C.; Cambau, E.; Daley, C.L.; Griffith, D.E.; Guglielmetti, L.; Holland, S.M.; Huitt, G.A.; et al. Treatment outcome definitions in nontuberculous mycobacterial pulmonary disease: An NTM-NET con-sensus statement. Eur. Respir. Soc. 2018, 51, 1800170. [CrossRef]

7. Griffith, D.E.; Aksamit, T.R. Therapy of refractory nontuberculous mycobacterial lung disease. Curr. Opin. Infect. Dis. 2012, 25, 218-227. [CrossRef]

8. Kwon, Y.-S.; Koh, W.-J.; Daley, C.L. Treatment ofMycobacterium aviumComplex Pulmonary Disease. Tuberc. Respir. Dis. 2019, 82, 15-26. [CrossRef]

9. Daley, C.L.; Iaccarino, J.M.; Lange, C.; Cambau, E.; Wallace, R.J., Jr.; Andrejak, C.; Böttger, E.C.; Brozek, J.; Griffith, D.E.; Guglielmetti, L.; et al. Treatment of nontuberculous mycobacterial pulmonary disease: An official ATS/ERS/ESCMID/IDSA clinical practice guideline. Clin. Infect. Dis. 2020, 71, e1-e36. [CrossRef]

10. Pidsley, R.; Zotenko, E.; Peters, T.J.; Lawrence, M.G.; Risbridger, G.P.; Molloy, P.; Van Djik, S.; Muhlhausler, B.; Stirzaker, C.; Clark, S.J. Critical evaluation of the Illumina MethylationEPIC BeadChip microarray for whole-genome DNA methylation profiling. Genome Biol. 2016, 17, 208. [CrossRef]

11. Dinardo, A.R.; Rajapakshe, K.; Nishiguchi, T.; Grimm, S.L.; Mtetwa, G.; Dlamini, Q.; Kahari, J.; Mahapatra, S.; Kay, A.W.; Maphalala, G.; et al. DNA hypermethylation during tuberculosis dampens host immune responsiveness. J. Clin. Investig. 2020, 130, 3113-3123. [CrossRef]

12. Shell, S.S.; Prestwich, E.G.; Baek, S.-H.; Shah, R.R.; Sassetti, C.M.; Dedon, P.; Fortune, S.M. DNA Methylation Impacts Gene Expression and Ensures Hypoxic Survival of Mycobacterium tuberculosis. PLoS Pathog. 2013, 9, e1003419. [CrossRef]

13. Heyn, H.; Esteller, M. DNA methylation profiling in the clinic: Applications and challenges. Nat. Rev. Genet. 2012, 13, 679-692. [CrossRef]

14. Mansell, G.; Gorrie-Stone, T.J.; Bao, Y.; Kumari, M.; Schalkwyk, L.S.; Mill, J.; Hannon, E. Guidance for DNA methylation studies: Statistical insights from the Illumina EPIC array. BMC Genom. 2019, 20, 1-15. [CrossRef]

15. Bansal, A.; Pinney, S.E. DNA methylation and its role in the pathogenesis of diabetes. Pediatr. Diabetes 2017, 18, 167-177. [CrossRef]

16. Samblas, M.; Milagro, F.I.; Martínez, A. DNA methylation markers in obesity, metabolic syndrome, and weight loss. Epigenetics 2019, 14, 421-444. [CrossRef]

17. Ovenden, E.S.; McGregor, N.W.; Emsley, R.A.; Warnich, L. DNA methylation and antipsychotic treatment mechanisms in schizophrenia: Progress and future directions. Prog. Neuro-Psychopharmacol. Biol. Psychiatry 2018, 81, 38-49. [CrossRef] [PubMed]

18. Montrose, L.; Ward, T.J.; Semmens, E.O.; Cho, Y.H.; Brown, B.; Noonan, C.W. Dietary intake is associated with respiratory health outcomes and DNA methylation in children with asthma. Allergy Asthma Clin. Immunol. 2017, 13, 12. [CrossRef] [PubMed] 
19. Wei, M.; Wang, L.; Wu, T.; Xi, J.; Han, Y.; Yang, X.; Zhang, D.; Fang, Q.; Tang, B. NLRP3 Activation Was Regulated by DNA Methylation Modification duringMycobacterium tuberculosisInfection. BioMed Res. Int. 2016, 2016, 1-10. [CrossRef] [PubMed]

20. Shu, C.-C.; Pan, S.-W.; Feng, J.-Y.; Wang, J.-Y.; Chan, Y.-J.; Yu, C.-J.; Su, W.-J. The Clinical Significance of Programmed Death-1, Regulatory T Cells and Myeloid Derived Suppressor Cells in Patients with Nontuberculous Mycobacteria-Lung Disease. J. Clin. Med. 2019, 8, 736. [CrossRef]

21. Iwata, K.; Oka, S.; Tsuno, H.; Furukawa, H.; Shimada, K.; Hashimoto, A.; Komiya, A.; Tsuchiya, N.; Katayama, M.; Tohma, S. Biomarker for nontuberculous mycobacterial pulmonary disease in patients with rheumatoid arthritis: Anti-glycopeptidolipid core antigen immunoglobulin A antibodies. Mod. Rheumatol. 2018, 28, 271-275. [CrossRef]

22. Griffith, D.E.; Aksamit, T.; Brown-Elliott, B.A.; Catanzaro, A.; Daley, C.; Gordin, F.; Holland, S.M.; Horsburgh, R.; Huitt, G.; Iademarco, M.F.; et al. An official ATS/IDSA statement: Diagnosis, treatment, and prevention of nontuberculous mycobacterial diseases. Am. J. Respir. Crit. Care Med. 2007, 175, 367-416. [CrossRef]

23. Kim, Y.; Han, B.G.; KoGES Group. Cohort profile: The Korean genome and epidemiology study (KoGES) consortium. Int. J. Epidemiol. 2017, 46, e20. [CrossRef]

24. Ulgen, E.; Ozisik, O.; Sezerman, O.U. pathfindR: An R Package for Comprehensive Identification of Enriched Pathways in Omics Data Through Active Subnetworks. Front. Genet. 2019, 10, 858. [CrossRef]

25. Shen, H.; Chen, Z.W. The crucial roles of Th17-related cytokines/signal pathways in M. tuberculosis infection. Cell. Mol. Immunol. 2018, 15, 216-225. [CrossRef]

26. Jo, E.-K.; Silwal, P.; Yuk, J.-M. AMPK-Targeted Effector Networks in Mycobacterial Infection. Front. Microbiol. 2019, 10, 520. [CrossRef]

27. Lachmandas, E.; Beigier-Bompadre, M.; Cheng, S.C.; Kumar, V.; van Laarhoven, A.; Wang, X.; Ammerdorffer, A.; Boutens, L.; de Jong, D.; Kanneganti, T.D.; et al. Rewiring cellular metabolism via the AKT/mTOR pathway contributes to host de-fence against Mycobacterium tuberculosis in human and murine cells. Eur. J. Immunol. 2016, 46, 2574-2586. [CrossRef]

28. Fang, F.; Wang, Y.; Li, R.; Zhao, Y.; Guo, Y.; Jiang, M.; Sun, J.; Ma, Y.; Ren, Z.; Tian, Z.; et al. Transcription factor E2F1 sup-presses dendritic cell maturation. J. Immunol. 2010, 184, 6084-6091. [CrossRef]

29. Villaseñor, T.; Madrid-Paulino, E.; Maldonado-Bravo, R.; Urbán-Aragón, A.; Pérez-Martínez, L.; Pedraza-Alva, G. Activation of the Wnt Pathway by Mycobacterium tuberculosis: A Wnt-Wnt Situation. Front. Immunol. 2017, 8, 50. [CrossRef]

30. Brandenburg, J.; Reiling, N. The Wnt Blows: On the functional role of Wnt signaling in Mycobacterium tuberculosis infection and beyond. Front. Immunol. 2016, 7. [CrossRef]

31. Lam, A.P.; Herazo-Maya, J.D.; Sennello, J.A.; Flozak, A.S.; Russell, S.; Mutlu, G.M.; Budinger, G.R.S.; DasGupta, R.; Varga, J.; Kaminski, N.; et al. Wnt CoreceptorLrp5Is a Driver of Idiopathic Pulmonary Fibrosis. Am. J. Respir. Crit. Care Med. 2014, 190, 185-195. [CrossRef]

32. Lee, S.W.; Wu, L.S.H.; Huang, G.M.; Huang, K.Y.; Lee, T.Y.; Weng, J.T.Y. Gene expression profiling identifies candidate bi-omarkers for active and latent tuberculosis. BMC Bioinform. 2016, 17, 27-39. [CrossRef]

33. Han, S.A.; Jhun, B.W.; Kim, S.Y.; Moon, S.M.; Yang, B.; Kwon, O.J.; Daley, C.L.; Shin, S.J.; Koh, W.J. miRNA expression pro-files and potential as biomarkers in nontuberculous mycobacterial pulmonary disease. Sci. Rep. 2020, 10, 1-13.

34. Jati, S.; Sarraf, T.R.; Naskar, D.; Sen, M. Wnt Signaling: Pathogen Incursion and Immune Defense. Front. Immunol. 2019, $10,2551$. [CrossRef]

35. Aung, H.; Sherman, J.; Tary-Lehman, M.; Toossi, Z. Analysis of transforming growth factor-beta 1 (TGF- $\beta 1$ ) expression in human monocytes infected with Mycobacterium avium at a single cell level by ELISPOT assay. J. Immunol. Methods 2002, 259, 25-32. [CrossRef]

36. Sheppard, D. Transforming growth factor $\beta$ : A central modulator of pulmonary and airway inflammation and fibrosis. Proc. Am. Thorac. Soc. 2006, 3, 413-417. [CrossRef] [PubMed]

37. Bermudez, L.E. Production of transforming growth factor-beta by Mycobacterium avium-infected human macrophages is as-sociated with unresponsiveness to IFN-gamma. J. Immunol. 1993, 150, 1838-1845.

38. Wang, J.; Yao, Y.; Xiong, J.; Wu, J.; Tang, X.; Li, G. Evaluation of the Inflammatory Response in Macrophages Stimulated with Exosomes Secreted byMycobacterium avium-Infected Macrophages. BioMed Res. Int. 2015, 2015, 1-9. [CrossRef]

39. Matsuyama, M.; Ishii, Y.; Yageta, Y.; Ohtsuka, S.; Ano, S.; Matsuno, Y.; Morishima, Y.; Yoh, K.; Takahashi, S.; Ogawa, K.; et al. Role of Th1/Th17 Balance Regulated by T-bet in a Mouse Model of Mycobacterium avium Complex Disease. J. Immunol. 2014, 192, 1707-1717. [CrossRef]

40. Matsuyama, M.; Ishii, Y.; Sakurai, H.; Ano, S.; Morishima, Y.; Yoh, K.; Takahashi, S.; Ogawa, K.; Hizawa, N. Overexpression of ROR $\gamma t$ Enhances Pulmonary Inflammation after Infection with Mycobacterium Avium. PLoS ONE 2016, 11, e0147064. [CrossRef] [PubMed]

41. Kim, S.Y.; Koh, W.J.; Park, H.; Jeon, K.; Kwon, O.; Cho, S.N.; Shin, S.J. Changes in serum immunomolecules during antibiotic therapy for Mycobacterium avium complex lung disease. Clin. Exp. Immunol. 2014, 176, 93-101. [CrossRef] [PubMed]

42. Philley, J.V.; Hertweck, K.L.; Kannan, A.; Brown-Elliott, B.A.; Wallace, R.J.; Kurdowska, A.; Ndetan, H.; Singh, K.P.; Miller, E.J.; Griffith, D.E.; et al. Sputum Detection of Predisposing Genetic Mutations in Women with Pulmonary Nontuberculous Mycobacterial Disease. Sci. Rep. 2018, 8, 11336. [CrossRef] [PubMed] 